\title{
Cooperative Diversity with Opportunistic Relaying
}

\author{
Aggelos Bletsas, Hyundong Shin, Moe Z. Win, and Andrew Lippman \\ Massachusetts Institute of Technology \\ 77 Massachusetts Avenue, Cambridge, MA 02139
}

\begin{abstract}
In this paper, we present single-selectionopportunistic-relaying with decode-and-forward (DaF) and amplify-and-forward ( $\mathrm{AaF})$ protocols under an aggregate power constraint. We show that opportunistic DaF relaying is equivalent to the outage bound of the optimal DaF strategy using all potential relays. We further show that opportunistic AaF relaying is outage-optimal with single-relay selection and significantly outperforms an AaF strategy with multiple-relay (MR) transmissions, in the presence of limited channel knowledge. These findings reveal that cooperative diversity benefits (under an aggregate power constraint) are useful even when cooperative relays choose not to transmit but rather choose to cooperatively listen; they act as passive relays and give priority to the transmission of a single opportunistic relay.
\end{abstract}

\section{INTRODUCTION}

Utilization of terminals distributed in space can significantly improve the performance of wireless communication [1]-[3]. For example, a pair of neighboring nodes with channel state information (CSI) can cooperatively beamform towards the final destination, increasing total capacity [2]. Even when CSI is not available or when radio hardware cannot support beamforming, cooperation between the source and a single relay provides improved robustness to wireless fading [3]. Basic results for cooperation are presented in [4]-[6] and references therein.

Scaling cooperation to more than one relay is still an open area of research, despite the recent interest in cooperative communication. One possible approach is the use of distributed space-time coding among participating nodes [7]. In practice, such code design is quite difficult due to the distributed and ad-hoc nature of cooperative links, as opposed to co-located multiple-input multiple-output (MIMO) systems. For example, it is impractical for each relay to acquire CSI about other relays (as needed in [8]) or for the destination to acquire CSI between the source and all relays. Hence, those channel states need to be communicated to each relay or the destination. Moreover, the number of useful antennas (distributed relays) for cooperation is generally unknown and varying. Therefore, coordination among the cooperating nodes is needed prior to a specific space-time coding scheme, designed for a fixed number of transmit antennas. Furthermore, it is often assumed in the literature that the superposition of signals transmitted by several relays is always constructive. ${ }^{1}$ Such assumption requires distributed phased-array techniques (beamforming) and unconventional radios with increased complexity and cost

\footnotetext{
${ }^{1}$ This case includes Gaussian relay channels where propagation coefficients are assumed to be real numbers [9].
}

of each transmitter. Finally, coherent reception of multiplerelay (MR) transmissions requires tracking of carrier phase differences among several transmit-receive pairs, which increases the cost of the receiver.

Therefore, simplification of radio hardware in cooperative diversity setups is important. Antenna selection, invented for classical multiple-antenna communications [10]-[12], is one approach to minimize the required cooperation overhead and to simultaneously realize the potential benefits of cooperation between multiple relays. In particular, a simple, distributed, single-relay selection algorithm was proposed for slow fading wireless environments [13]. This single-relay selection provides no performance loss from a diversity-multiplexing gain tradeoff perspective, compared to schemes that rely on distributed space-time coding [14]. Each intermediate relay overhears the transmission of pilot signals between the source and destination, and evaluates its own end-to-end channel quality. The relay that maximizes a function of its channel quality, towards the source and destination, is selected in a distributed manner. The main idea of [13] is that once a race condition among all relays is introduced, individual relays do not need to acquire CSI about the links of other relays; instead, their own CSI towards the source and destination would suffice.

In this paper, we present single-selection-opportunisticrelaying with decode-and-forward $(\mathrm{DaF})$ and amplify-andforward $(\mathrm{AaF})$ protocols and analyze its outage probability under a source power constraint

$$
\mathcal{P}_{\text {source }}=\zeta \mathcal{P}_{\text {tot }}
$$

and an aggregate relay power constraint

$$
\mathcal{P}_{\text {relay }}=\sum_{k=1}^{K} \mathcal{P}_{k}=(1-\zeta) \mathcal{P}_{\text {tot }}
$$

where $K$ is the number of relays, $\mathcal{P}_{\text {tot }}$ is the total end-to-end (i.e., source-relay-destination) transmission power, $\mathcal{P}_{\text {source }}$ is the transmission power of the source, $\mathcal{P}_{k}, k=1, \ldots, K$, is the transmission power of the $k$ th relay, and $\mathcal{P}_{\text {relay }}$ is the aggregate relay power allocated to the set $\mathcal{S}_{\text {relay }}=\{1,2, \ldots, K\}$ of $K$ relays. Note that $\zeta \in(0,1]$ and $(1-\zeta) \in[0,1)$ denote the fractions of the total end-to-end power $\mathcal{P}_{\text {tot }}$ allocated to the source transmission and overall relay transmission, respectively.

The motivation behind imposing the aggregate power constraints (1) and (2) is threefold: (i) transmission power is a network resource that affects both the life time of the 
network with battery-operated terminals and the scalability of the network; (ii) regulatory agencies may limit total transmission power due to the fact that each transmission can cause interference to the others in the network; and (iii) cooperative diversity benefits can be exploited even when relays do not transmit (and therefore, do not add transmission energy into the network). We show that opportunistic $\mathrm{DaF}$ relaying is equivalent to the outage bound of the optimal DaF strategy using all potential relays. We further show that opportunistic AaF relaying is outage-optimal with single-relay selection and significantly outperforms an $\mathrm{AaF}$ strategy with multiplerelay (MR) transmissions, in the presence of limited channel knowledge. These observations reveal that relays are useful even when they do not actively transmit, provided that they adhere to the "opportunistic" cooperation rule and give priority to the "best" available relay. The simplicity of our scheme allows immediate implementation in a custom radio hardware. An implementation example can be found in [13].

\section{Models AND PRotocols}

We consider a half-duplex dual-hop communication scenario in a cluttered environment depicted in Fig. 1, where the direct path between the source and destination is blocked by an intermediate wall, while relays are located at the periphery of the obstacle (around-the-corner). The relays can communicate with both endpoints (source and destination). During the first hop, the source (without any CSI) transmits $N / 2$ symbols and the relays listen, while during the second hop, the relays forward a version of the received signal using the same number of symbols. ${ }^{2}$ The channel is assumed to remain constant during the two hops (at least $N$-symbol coherence time) with Rayleigh fading.

The received signal in a link $(\mathrm{A} \rightarrow \mathrm{B})$ between two nodes " $\mathrm{A}$ " and " $\mathrm{B}$ " is given by

$$
y_{\mathrm{B}}=\alpha_{\mathrm{AB}} x_{\mathrm{A}}+n_{\mathrm{B}}
$$

where $x_{\mathrm{A}}$ is the signal transmitted at the node $\mathrm{A}, \alpha_{\mathrm{AB}} \sim$ $\mathcal{C N}\left(0, \Omega_{\mathrm{AB}}\right)$ is the channel gain between the link $\mathrm{A} \rightarrow \mathrm{B}$, and $n_{\mathrm{B}} \sim \mathcal{C N}\left(0, N_{0}\right)$ is the additive white Gaussian noise (AWGN) at the node B. ${ }^{3}$ For each link, let $\gamma_{\mathrm{AB}} \triangleq\left|\alpha_{\mathrm{AB}}\right|^{2}$ be the instantaneous squared channel strength, which obeys a statistically independent exponential distribution with hazard rate $1 / \Omega_{\mathrm{AB}}$, denoted by $\gamma_{\mathrm{AB}} \sim \Upsilon\left(1 / \Omega_{\mathrm{AB}}\right)$. The probability density function (p.d.f.) of $\gamma_{\mathrm{AB}}$ is given by

$$
p_{\gamma_{\mathrm{AB}}}(x)=\frac{1}{\Omega_{\mathrm{AB}}} \exp \left(-x / \Omega_{\mathrm{AB}}\right), \quad x \geq 0 .
$$

If the node $\mathrm{A}$ is the source, then $\mathbb{E}\left\{\left|x_{\mathrm{A}}\right|^{2}\right\}=\mathcal{P}_{\text {source }}$. Similarly, if the node A is the $k$ th relay, then $\mathbb{E}\left\{\left|x_{\mathrm{A}}\right|^{2}\right\}=\mathcal{P}_{k}$. Specifically, for each relay $k \in \mathcal{S}_{\text {relay }}$, we designate a link from

\footnotetext{
${ }^{2}$ If the source is allowed to transmit different symbols during the second hop, one channel degree of freedom would not be wasted and the spectral efficiency can be improved [15], [16]. However, in this paper, we are interested in finding the optimal strategy for relay transmissions and hence, simplify their operation.

${ }^{3} \mathcal{C N}\left(\mu, \sigma^{2}\right)$ denotes a complex circularly symmetric Gaussian distribution with mean $\mu$ and variance $\sigma^{2}$.
}
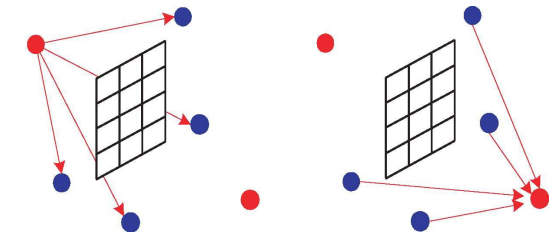

Fig. 1. A half-duplex dual-hop communication scenario: the source and destination are blocked or have poor connection. During the first hop, the source transmits and relays listen, while during the second hop, the relays transmit and the destination listens.

the source to the $k$ th relay by $\mathrm{S} \rightarrow k$ and a link from the $k$ th relay to the destination by $k \rightarrow \mathrm{D}$. Also, $R$ denotes the end-toend spectral efficiency in bps/Hz and SNR $\triangleq \mathcal{P}_{\text {tot }} / N_{0}$ denotes the end-to-end transmit SNR. For the links $\mathrm{S} \rightarrow k$ and $k \rightarrow \mathrm{D}$, the average received SNRs are equal to $\eta_{\mathrm{S} k} \triangleq \Omega_{\mathrm{S} k} \mathcal{P}_{\text {source }} / N_{0}$ and $\eta_{k \mathrm{D}} \triangleq \Omega_{k \mathrm{D}} \mathcal{P}_{k} / N_{0}$, respectively.

To minimize overhead and simplify protocol implementation, cooperation is coordinated only every $N$ symbols. We consider two modes of coordination: (i) reactive coordination among $\mathrm{DaF}$ relays and (ii) proactive coordination among $\mathrm{DaF}$ or AaF relays. In a reactive mode, relays that successfully decode the message participate in cooperation, whereas in a proactive mode, specific relays that are selected prior to the source transmission participate in cooperation.

It should be noted that the optimal power allocation across the source and relays depends on CSI knowledge and can be $\mathcal{P}_{\text {source }} \neq \mathcal{P}_{\text {relay }}$ [17]. However, this is operational when (i) global CSI about the whole network (including channel states between the relays and destination) is available at the source or (ii) there exists a good direct link between the source and destination. None of these conditions are applicable to our study. In fact, our main focus is not just optimal power allocation but a more general question of what relays should do optimally-re-transmit or not.

\section{DECODE-AND-FORWARD RELAYING}

\section{A. Reactive DaF}

In a reactive $\mathrm{DaF}$ scheme, the relays that successfully decode the message regenerate and transmit it, possibly through a distributed space-time code [7].

1) Reactive Multiple-Relay DaF: The MR transmission during the second hop is performed only by the subset $\mathcal{D}_{K}$ of $K$ relays, defined by

$$
\mathcal{D}_{K} \triangleq\left\{k \in \mathcal{S}_{\text {relay }}: \frac{1}{2} \log _{2}\left(1+\zeta \mathrm{SNR} \cdot \gamma_{\mathrm{S} k}\right) \geq R\right\}
$$

where the decoding at the relays is assumed be successful if no outage event happens during the first hop [3], [7]. Since communication happens in two half-duplex hops, the required spectral efficiency per hop is equal to $2 R$ so that the endto-end spectral efficiency is $R$, which is comparable to direct non-cooperative communication. 
Let $\mathcal{D}_{K}(\ell) \subseteq \mathcal{S}_{\text {relay }}$ be a decoding subset with $\ell$ relays (i.e., cardinality $\left|\mathcal{D}_{K}(\ell)\right|=\ell$ ). Then, we have

$$
\begin{aligned}
\operatorname{Pr}\left\{\mathcal{D}_{K}(\ell)\right\} & =\prod_{i \in \mathcal{D}_{K}(\ell)} \operatorname{Pr}\left\{\gamma_{\mathrm{Si}} \geq \kappa_{1}\right\} \prod_{j \notin \mathcal{D}_{K}(\ell)} \operatorname{Pr}\left\{\gamma_{\mathrm{S} j} \leq \kappa_{1}\right\} \\
& =\prod_{i \in \mathcal{D}_{K}(\ell)} e^{-\frac{\kappa_{1}}{\Omega_{\mathrm{Si} i}}} \prod_{j \notin \mathcal{D}_{K}(\ell)}\left(1-e^{-\frac{\kappa_{1}}{\Omega_{\mathrm{S} j}}}\right)
\end{aligned}
$$

where $\kappa_{1}=\frac{2^{2 R}-1}{\zeta \mathrm{SNR}}$. The outage probability for reactive MR $\mathrm{DaF}$ can be bounded as

$$
\begin{aligned}
& P_{\text {MR-DaF }}^{\text {(react) }} \text { (outage) } \\
& \quad \leq \sum_{\ell=0}^{K} \sum_{\mathcal{D}_{K}(\ell)} \operatorname{Pr}\left\{\text { outage } \mid \mathcal{D}_{K}(\ell)\right\} \operatorname{Pr}\left\{\mathcal{D}_{K}(\ell)\right\}
\end{aligned}
$$

where the second summation is over all $\left(\begin{array}{c}K \\ \ell\end{array}\right)$ different decoding subsets with exactly $\ell$ successfully decodable relays. In (7), the conditional outage probability is given by

$$
\begin{aligned}
& \operatorname{Pr}\left\{\text { outage } \mid \mathcal{D}_{K}(\ell)\right\} \\
& =\operatorname{Pr}\left\{\frac{1}{2} \log _{2}\left(1+\sum_{k \in \mathcal{D}_{K}(\ell)} \frac{\mathcal{P}_{k} \gamma_{k \mathrm{D}}}{N_{0}}\right)<R\right\}
\end{aligned}
$$

with $\sum_{k \in \mathcal{D}_{K}(\ell)} \mathcal{P}_{k}=\mathcal{P}_{\text {relay }}$. Note that there are $2^{K}$ possible decoding subsets for $K$ relays, including $\mathcal{D}_{K}(0)$, i.e., the set with no decodable relay during the first hop of the protocol.

Let $\mathcal{A}_{\mathcal{D}_{K}(\ell)}$ be the $\ell \times \ell$ diagonal matrix with elements $\left\{\eta_{k \mathrm{D}}\right\}_{k \in \mathcal{D}_{K}(\ell)}$ and $\varphi_{i}$ be its $i$ th diagonal element. Then, we have

$$
\begin{aligned}
& \operatorname{Pr}\left\{\text { outage } \mid \mathcal{D}_{K}(\ell)\right\} \\
& \quad=1-\sum_{i=1}^{\varrho} \sum_{j=1}^{\vartheta_{i}} \sum_{k=0}^{j-1} \frac{\mathcal{X}_{i, j}\left(\mathcal{A}_{\mathcal{D}_{K}(\ell)}\right)}{k !}\left(\frac{2^{2 R}-1}{\varphi_{\langle i\rangle}}\right)^{k} e^{-\frac{2^{2 R}-1}{\varphi_{i i\rangle}}}
\end{aligned}
$$

where $\varrho$ is the number of distinct diagonal elements of $\mathcal{A}_{\mathcal{D}_{K}(\ell)}, \varphi_{\langle 1\rangle}>\varphi_{\langle i\rangle}>\ldots>\varphi_{\langle\varrho\rangle}$ are the distinct diagonal elements in decreasing order, $\vartheta_{i}$ is the multiplicity of $\varphi_{\langle i\rangle}$, and $\mathcal{X}_{i, j}\left(\mathcal{A}_{\mathcal{D}_{K}(\ell)}\right)$ is the $(i, j)$ th characteristic coefficient of $\mathcal{A}_{\mathcal{D}_{K}(\ell)}$ [18, Definition 6]. Combining (6), (7), and (9), we can obtain the upper bound on the outage probability for reactive MR DaF relaying.

2) Reactive Opportunistic DaF: For opportunistic relaying, the "best" relay $b_{\mathrm{DaF}}^{*}$ among the $\ell$ relays in the decoding subset $\mathcal{D}_{K}(\ell)$ is chosen naturally to maximize the instantaneous channel strength between the links $k \rightarrow \mathrm{D}$ for all $k \in \mathcal{D}_{K}(\ell)$ :

$$
b_{\mathrm{DaF}}^{*}=\arg \max _{k \in \mathcal{D}_{K}(\ell)} \gamma_{k \mathrm{D}} .
$$

This opportunistic relay selection yields

$$
\begin{aligned}
\sum_{k \in \mathcal{D}_{K}(\ell)} \frac{\mathcal{P}_{k} \gamma_{k \mathrm{D}}}{N_{0}} & \leq \sum_{k \in \mathcal{D}_{K}(\ell)} \frac{\mathcal{P}_{k} \gamma_{b_{\mathrm{DaF}}^{*} \mathrm{D}}}{N_{0}} \\
& =\gamma_{b_{\mathrm{DaF}}^{*} \mathrm{D}} \cdot(1-\zeta) \mathrm{SNR}
\end{aligned}
$$

and minimizes the conditional outage probability in (8) as

$$
\begin{aligned}
\operatorname{Pr} & \left\{\text { outage } \mid \mathcal{D}_{K}(\ell)\right\} \\
& \geq \operatorname{Pr}\left\{\frac{1}{2} \log _{2}\left(1+(1-\zeta) \text { SNR } \max _{k \in \mathcal{D}_{K}(\ell)} \gamma_{k \mathrm{D}}\right)<R\right\} \\
& =\prod_{k \in \mathcal{D}_{K}(\ell)} \operatorname{Pr}\left\{\gamma_{k \mathrm{D}}<\kappa_{2}\right\}
\end{aligned}
$$

where $\kappa_{2}=\frac{2^{2 R}-1}{(1-\zeta) \mathrm{SNR}}$. Note that (12) states simply that if the "best" relay fails, then all relays in $\mathcal{D}_{K}(\ell)$ should fail because the "best" relay has the strongest path $\gamma_{b_{\text {DaF }}^{*}}$ between the links $k \rightarrow \mathrm{D}$ for all $k \in \mathcal{D}_{K}(\ell)$. The minimization of (12) holds for any power allocation $\zeta$. For quasi-static fading environments, a simple method can be devised to select the relay with the maximum channel strength $\gamma_{b_{\mathrm{DaF}}^{*} \mathrm{D}}$ in a distributed manner similar to the work in [13], [14].

Using (6) and (7) in conjunction with (12) for the opportunistic relay-selection rule (10), we obtain the upper bound on the outage probability for reactive opportunistic $\mathrm{DaF}$ relaying as

$$
\begin{array}{r}
P_{\text {Opp-DaF }}^{\text {(react) }} \text { (outage) } \leq \sum_{\ell=0}^{K} \sum_{\mathcal{D}_{K}(\ell)}\left[\prod_{i \in \mathcal{D}_{K}(\ell)} e^{-\frac{\kappa_{1}}{\Omega_{\mathrm{S} i}}}\left(1-e^{-\frac{\kappa_{2}}{\Omega_{i \mathrm{D}}}}\right)\right. \\
\left.\times \prod_{j \notin \mathcal{D}_{K}(\ell)}\left(1-e^{-\frac{\kappa_{1}}{\Omega_{\mathrm{S} j}}}\right)\right] \\
=\prod_{k=1}^{K}\left[1-e^{-\frac{2^{2 R}-1}{\mathrm{SNR}}\left(\frac{1}{\zeta \Omega_{\mathrm{S} k}}+\frac{1}{(1-\zeta) \Omega_{k \mathrm{D}}}\right)}\right]
\end{array}
$$

where the last equality follows from the multinomial equality

$$
\begin{aligned}
& \prod_{k=1}^{K}\left(1-a_{k} b_{k}\right)=\prod_{k=1}^{K}\left[a_{k}\left(1-b_{k}\right)+\left(1-a_{k}\right)\right] \\
& =\sum_{\ell=0}^{K} \sum_{\substack{\mathcal{S}_{\ell} \subseteq\{1,2, \ldots, K\} \\
\left|\mathcal{S}_{\ell}\right|=\ell}}\left[\prod_{i \in \mathcal{S}_{\ell}} a_{i}\left(1-b_{i}\right) \prod_{j \notin \mathcal{S}_{\ell}}\left(1-a_{i}\right)\right] .
\end{aligned}
$$

Note that (13) implies that the outage event happens only when all relays are in outage.

\section{B. Proactive Opportunistic DaF}

In proactive opportunistic relaying, the "best" relay $b_{\mathrm{DaF}}^{*}$ is chosen prior to the source transmission among a collection of $K$ possible candidates in a distributed fashion that requires each relay to know its own instantaneous signal strength (but not phase) between the links $\mathrm{S} \rightarrow k$ and $k \rightarrow \mathrm{D}$ ( $k \in \mathcal{S}_{\text {relay }}$ ). The relay selection completes within a fraction of the channel coherence time and the selected single relay is then used for information relaying. A method of distributed timers allows to select the "best" relay without CSI about the links of other relays. The "best" relay $b_{\mathrm{DaF}}^{*}$ is chosen to maximize the minimum of the weighted channel strengths between the links 
$\mathrm{S} \rightarrow k$ and $k \rightarrow \mathrm{D}$ for all $k \in \mathcal{S}_{\text {relay }}{ }^{4}$

$$
b_{\mathrm{DaF}}^{*}=\arg \max _{k \in \mathcal{S}_{\text {relay }}} \min \left\{\zeta \gamma_{\mathrm{S} k},(1-\zeta) \gamma_{k \mathrm{D}}\right\} .
$$

In this case, communication through the "best" opportunistic relay fails due to outage when the following event happens:

$$
\begin{aligned}
& \left\{\frac{1}{2} \log _{2}\left(1+\zeta \mathrm{SNR} \cdot \gamma_{\mathrm{S} b_{\text {DaF }}^{*}}\right)<R\right\} \\
& \bigcup\left\{\frac{1}{2} \log _{2}\left(1+(1-\zeta) \mathrm{SNR} \cdot \gamma_{b_{\text {DaF }}^{*} \mathrm{D}}\right)<R\right\}
\end{aligned}
$$

or equivalently,

$$
\left\{\gamma_{\mathrm{S} b_{\mathrm{DaF}}^{*}}<\kappa_{1}\right\} \bigcup\left\{\gamma_{b_{\mathrm{DaF}}^{*} \mathrm{D}}<\kappa_{2}\right\} .
$$

Note that (17) simply states that opportunistic relaying fails if either of the two hops (from the source to the best relay or from the best relay to the destination) fail.

Let $W_{k}^{(\mathrm{DaF})}=\min \left\{\zeta \gamma_{\mathrm{S} k},(1-\zeta) \gamma_{k \mathrm{D}}\right\}$. Then, we have

$$
W_{k}^{(\mathrm{DaF})} \sim \Upsilon\left(\frac{1}{\zeta \Omega_{\mathrm{Sk}}}+\frac{1}{(1-\zeta) \Omega_{k \mathrm{D}}}\right)
$$

which follows from the fact that the minimum of two independent exponential r.v.'s is again an exponential r.v. with a hazard rate equal to the sum of the two hazard rates. From (15), (17), and (18), we obtain the outage probability for proactive opportunistic $\mathrm{DaF}$ relaying as follows:

$$
\begin{aligned}
& P_{\mathrm{Opp}-\mathrm{DaF}}^{\text {proact }}(\text { outage })=\operatorname{Pr}\left\{\gamma_{\mathrm{Sb}_{\mathrm{DaF}}^{*}}<\kappa_{1} \bigcup \gamma_{b_{\mathrm{DaF}}^{*} \mathrm{D}}<\kappa_{2}\right\} \\
& =\operatorname{Pr}\left\{W_{b_{\mathrm{DaF}}^{*}}^{(\mathrm{DaF})}<\frac{2^{2 R}-1}{\mathrm{SNR}}\right\} \\
& =\operatorname{Pr}\left\{\max _{k \in \mathcal{S}_{\text {relay }}} W_{k}^{(\mathrm{DaF})}<\frac{2^{2 R}-1}{\mathrm{SNR}}\right\} \\
& =\prod_{k=1}^{K}\left[1-e^{-\frac{2^{2 R}-1}{\operatorname{SNR}}\left(\frac{1}{\zeta \Omega_{\mathrm{S} k}}+\frac{1}{(1-\zeta) \Omega_{k \mathrm{D}}}\right)}\right] \text {. }
\end{aligned}
$$

It is worth remarking that the outage probability (19) for the proactive opportunistic scheme agrees exactly with (13). Moreover, proactive coordination requires a smaller cooperation overhead in reception energy since all relays, except a single opportunistic relay, can enter an idle mode during the first hop of the protocol. Therefore, our proactive strategy can be viewed as energy-efficient routing in the network. In contrast, the reactive schemes require all relays to receive information during the first hop and therefore, cooperation overhead in reception energy scales proportionally with the network size. This overhead may not be negligible especially in battery-operated terminals, when strong forward error correction (which requires energy-expensive routines) is used.

\footnotetext{
${ }^{4}$ Instead of the minimum, the harmonic mean of two path strengths has been also considered in [13].
}

\section{AMPLIFY-AND-FORWARD RELAYING}

\section{A. Multiple-Relay AaF}

When no direct communication is available between the source and destination, the mutual information for the AaF strategy with $K$ relays subject to the power constraint (2) is given by

$\mathcal{I}_{\text {MR-AaF }}=\frac{1}{2} \log _{2}\left\{1+\frac{\mathcal{P}_{\text {source }}}{N_{0}} \frac{\left|\sum_{k=1}^{K} \sqrt{\frac{\mathcal{P}_{k}}{\Omega_{\mathrm{S} k} \mathcal{P}_{\text {source }}+N_{0}}} \alpha_{\mathrm{S} k} \alpha_{k \mathrm{D}}\right|^{2}}{\left(1+\sum_{k=1}^{K} \frac{\mathcal{P}_{k}\left|\alpha_{k \mathrm{D}}\right|^{2}}{\Omega_{\mathrm{S} k} \mathcal{P}_{\text {source }}+N_{0}}\right)}\right\}$.

Let $\mathbf{h}_{1} \sim \tilde{\mathcal{N}}_{K}\left(\mathbf{0}, \mathbf{I}_{K}\right)$ and $\mathbf{h}_{2} \sim \tilde{\mathcal{N}}_{K}\left(\mathbf{0}, \mathbf{I}_{K}\right)$ be independent complex $K$-dimensional (column) Gaussian vectors, where $\mathbf{I}_{K}$ is the $K \times K$ identity matrix. Then, defining $K \times K$ diagonal matrices

$$
\begin{aligned}
& \mathbf{G}_{1}=\operatorname{diag}\left(\sqrt{\eta_{\mathrm{S} 1}}, \sqrt{\eta_{\mathrm{S} 2}}, \ldots, \sqrt{\eta_{\mathrm{S} K}}\right) \\
& \mathbf{G}_{2}=\operatorname{diag}\left(\sqrt{\frac{\eta_{1 \mathrm{D}}}{\eta_{\mathrm{S} 1}+1}}, \sqrt{\frac{\eta_{2 \mathrm{D}}}{\eta_{\mathrm{S} 2}+1}}, \ldots, \sqrt{\frac{\eta_{K \mathrm{D}}}{\eta_{\mathrm{S} K}+1}}\right),
\end{aligned}
$$

we can rewrite (20) as

$$
\begin{aligned}
\mathcal{I}_{\text {MR-AaF }} & =\frac{1}{2} \log _{2}\left\{1+\frac{\left|\mathbf{h}_{2}^{\dagger} \mathbf{G}_{1} \mathbf{G}_{2} \mathbf{h}_{1}\right|^{2}}{1+\mathbf{h}_{2}^{\dagger} \mathbf{G}_{2}^{2} \mathbf{h}_{2}}\right\} \\
& =\frac{1}{2} \log _{2}\{1+\underbrace{\mathbf{h}_{1}^{\dagger} \boldsymbol{\Sigma}_{\left(\mathbf{h}_{2}\right)} \mathbf{h}_{1}}_{\triangleq \Xi}\}
\end{aligned}
$$

where $\dagger$ denotes the transpose conjugate and

$$
\boldsymbol{\Sigma}_{\left(\mathbf{h}_{2}\right)} \in \mathbb{C}^{K \times K}=\frac{\mathbf{G}_{2} \mathbf{G}_{1} \mathbf{h}_{2} \mathbf{h}_{2}^{\dagger} \mathbf{G}_{1} \mathbf{G}_{2}}{1+\mathbf{h}_{2}^{\dagger} \mathbf{G}_{2}^{2} \mathbf{h}_{2}} .
$$

Note that $\boldsymbol{\Sigma}_{\left(\mathbf{h}_{2}\right)}$ is of one rank and its nonzero eigenvalue $\lambda_{\boldsymbol{\Sigma}}$ is equal to

$$
\lambda_{\boldsymbol{\Sigma}}=\frac{\mathbf{h}_{2}^{\dagger}\left(\mathbf{G}_{1} \mathbf{G}_{2}\right)^{2} \mathbf{h}_{2}}{1+\mathbf{h}_{2}^{\dagger} \mathbf{G}_{2}^{2} \mathbf{h}_{2}} .
$$

Since the distribution of $\mathbf{h}_{1}$ is unitary invariant, i.e.,

$$
\mathbf{U h}_{1} \sim \tilde{\mathcal{N}}_{K}\left(\mathbf{0}, \mathbf{I}_{K}\right), \quad \forall \mathbf{U} \in\left\{\mathbf{U} \in \mathbb{C}^{K \times K}: \mathbf{U U}^{\dagger}=\mathbf{I}_{K}\right\}
$$

it is clear that $\Xi \mid \mathbf{h}_{2} \sim \Upsilon\left(1 / \lambda_{\boldsymbol{\Sigma}}\right)$ and hence, the outage probability for MR AaF relaying is given by

$$
\begin{aligned}
P_{\mathrm{MR}-\mathrm{AaF}}(\text { outage }) & =\operatorname{Pr}\left\{\mathcal{I}_{\mathrm{MR}-\mathrm{AaF}}<R\right\} \\
& =\mathbb{E}_{\mathbf{h}_{2}}\left\{F_{\Xi \mid \mathbf{h}_{2}}\left(2^{2 R}-1\right)\right\} \\
& =\mathbb{E}_{\lambda_{\boldsymbol{\Sigma}}}\left\{1-e^{-\frac{2^{2 R}-1}{\lambda_{\boldsymbol{\Sigma}}}}\right\} \\
& =1-\int_{0}^{\infty} e^{-\frac{2^{2 R}-1}{\lambda}} p_{\lambda_{\boldsymbol{\Sigma}}}(\lambda) d \lambda
\end{aligned}
$$

where $p_{\lambda_{\Sigma}}(\lambda)$ is the p.d.f. of $\lambda_{\Sigma}$ (we do not present its expression due to a space limit). 


\section{B. Opportunistic AaF}

From (20), we see that the maximum mutual information with a single-relay selection, i.e., the mutual information for opportunistic AaF relaying is

$$
\mathcal{I}_{\text {Opp-AaF }}=\max _{k \in \mathcal{S}_{\text {relay }}} \frac{1}{2} \log _{2}\left\{1+\frac{\frac{\mathcal{P}_{\text {source }}}{N_{0}} \gamma_{\mathrm{S} k} \gamma_{k \mathrm{D}}}{\frac{\zeta}{1-\zeta} \Omega_{\mathrm{S} k}+\frac{N_{0}}{\mathcal{P}_{\text {relay }}}+\gamma_{k \mathrm{D}}}\right\} \text {. }
$$

Hence, for opportunistic relaying, the "best" relay $b_{\mathrm{AaF}}^{*}$ among $K$ relays in $\mathcal{S}_{\text {relay }}$ is chosen proactively to maximize the mutual information (or to minimize the outage probability) as follows:

$$
b_{\mathrm{AaF}}^{*}=\arg \max _{k \in \mathcal{S}_{\text {relay }}} \frac{\gamma_{\mathrm{S} k} \gamma_{k \mathrm{D}}}{\frac{\zeta}{1-\zeta}\left(1+\frac{1}{\eta_{\mathrm{S} k}}\right) \mathbb{E}\left\{\gamma_{\mathrm{S} k}\right\}+\gamma_{k \mathrm{D}}} .
$$

Note that individual relays do not need to acquire CSI about the links of other relays and hence, the opportunistic relay (27) can be selected in a distributed manner [13], [14].

Let

$$
W_{k \in \mathcal{S}_{\text {relay }}}^{(\mathrm{AaF})}=\frac{\gamma_{\mathrm{S} k} \gamma_{k \mathrm{D}}}{\frac{\zeta}{1-\zeta}\left(1+\frac{1}{\eta_{\mathrm{sk}}}\right) \Omega_{\mathrm{S} k}+\gamma_{k \mathrm{D}}} .
$$

Then, the outage probability for opportunistic $\mathrm{AaF}$ relaying is given by

$$
\begin{aligned}
P_{\text {Opp-AaF }}(\text { outage }) & =\operatorname{Pr}\left\{W_{b_{\mathrm{AaF}}^{*}}^{(\mathrm{AaF})}<\kappa_{1}\right\} \\
& =\operatorname{Pr}\left\{\max _{k \in \mathcal{S}_{\text {relay }}} W_{k}^{(\mathrm{AaF})}<\kappa_{1}\right\} \\
& =\prod_{k=1}^{K}\left[1-\int_{0}^{\infty} \exp \left\{-z-\frac{2^{2 R}-1}{\eta_{\mathrm{s} k}}\right.\right. \\
& \left.\left.\times\left[1+\frac{\zeta}{1-\zeta} \frac{\Omega_{\mathrm{s} k}}{\Omega_{k \mathrm{D}}}\left(1+\frac{1}{\eta_{\mathrm{s} k}}\right) \frac{1}{z}\right]\right\} d z\right] .
\end{aligned}
$$

\section{Numerical RESUlts}

In this section, we give some numerical examples of the outage probability as a function of SNR with power allocation $\zeta=0.5$. The optimal power allocation $\zeta$ is feasible, only when the source has knowledge of the overall network topology in terms of the average channel gains $\Omega_{\mathrm{S} k}$ and $\Omega_{k \mathrm{D}}$ for all participating relays $k \in \mathcal{S}_{\text {relay }}$. However, since it requires a considerable overhead, no CSI at the source is assumed in practice. In this case, the equal-power allocation to the source and the best opportunistic relay, i.e., $\zeta=0.5$ is a natural choice. ${ }^{5}$

\section{A. Decode-and-Forward Relaying}

Fig. 2 shows the outage probability as a function of SNR for the $\mathrm{DaF}$ strategy with 6 relays $(K=6)$ at the end-to-end spectral efficiency $R=1 \mathrm{bps} / \mathrm{Hz}$ in symmetric channels with $\Omega_{\mathrm{S} k}=\Omega_{k \mathrm{D}}=1, k=1,2, \ldots, 6$. In this figure, we show the performance of (i) proactive opportunistic DaF relaying,

\footnotetext{
${ }^{5}$ Notably, an interesting question is how much performance loss is incurred from the use of this suboptimal power allocation $\zeta=0.5$. Due to a space limit, we do not address this issue in the paper.
}

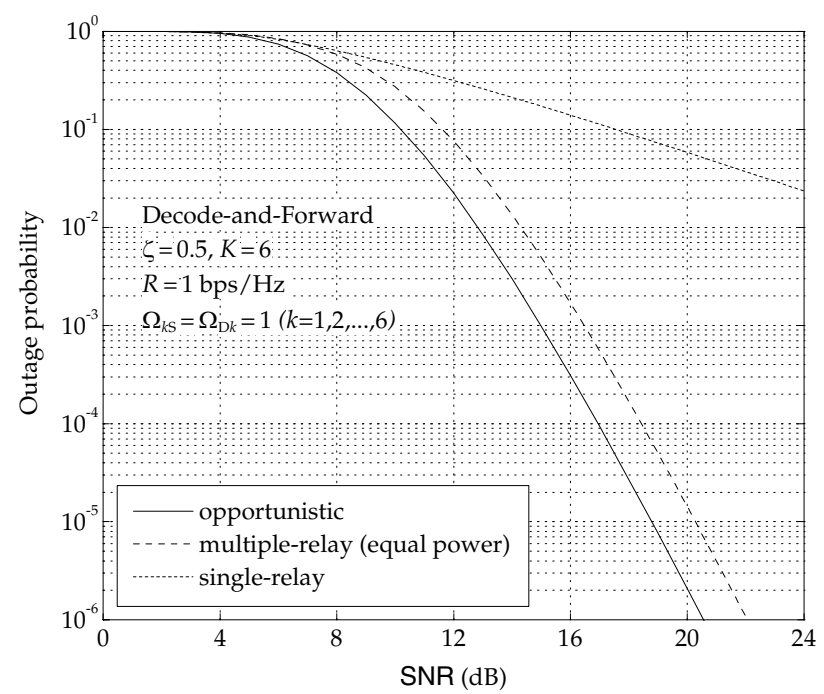

Fig. 2. Outage probability as a function of SNR for the DaF strategy at the end-to-end spectral efficiency $R=1 \mathrm{bps} / \mathrm{Hz}$ in symmetric channels. $\zeta=0.5, K=6$, and $\Omega_{\mathrm{S} k}=\Omega_{k \mathrm{D}}=1, k=1,2, \ldots, 6$. Opportunistic $\mathrm{DaF}$ relaying is compared with reactive $\mathrm{DaF}$ schemes with equal-power MR transmissions and single-relay selection with the maximum average channel gain $\max _{k \in \mathcal{D}_{K}(\ell)} \Omega_{k \mathrm{D}}$.

(ii) the upper bound for reactive $\mathrm{DaF}$ relaying with equalpower MR transmissions, and (iii) the upper bound for reactive $\mathrm{DaF}$ relaying via single-relay selection with the maximum average channel gain $\max _{k \in \mathcal{D}_{K}(\ell)} \Omega_{k \mathrm{D}}$. For the symmetric case, single-relay selection based on the average channel gains amounts to selecting just one successful relay randomly (since all relays in the decoding subset $\mathcal{D}_{K}(\ell)$ have the same mean channel gain to the destination) and transmitting with full relaying power $\mathcal{P}_{\text {relay }}$. Also, under limited channel knowledge at each relay, the optimal power allocation for MR DaF relaying is infeasible and equal power for the decoding subset $\mathcal{D}_{K}(\ell)$, i.e., $\mathcal{P}_{k}=\mathcal{P}_{\text {relay }} / \ell$ for all $k \in \mathcal{D}_{K}(\ell)$ in reactive $\mathrm{DaF}$ relaying is a reasonable solution.

It can be seen that despite its simplicity, opportunistic relaying provides a gain in SNR on the order of $2 \mathrm{~dB}$ relative to MR $\mathrm{DaF}$ relaying. This finding reveals that cooperative diversity gains do not necessarily arise from simultaneous transmissions but instead, resilience to fading arises from the availability of several potential paths towards the destination. It is therefore useful to select the best one. The main difficulty here is to have the network as a whole entity cooperate in order to rapidly discover the best path with minimal overhead. Ideas on how such selection can be performed in a distributed manner were demonstrated in [13] for slow fading environments. In contrast to single opportunistic relay selection, a single-relay selection based on average channel gains incurs a substantial penalty loss. This is due to the fact that selecting a relay with average channel gains removes potential selection diversity benefits.

\section{B. Amplify-and-Forward Relaying}

Fig. 3 shows the outage probability as a function of SNR for the AaF strategy with 6 relays $(K=6)$ at $R=1 \mathrm{bps} / \mathrm{Hz}$ 


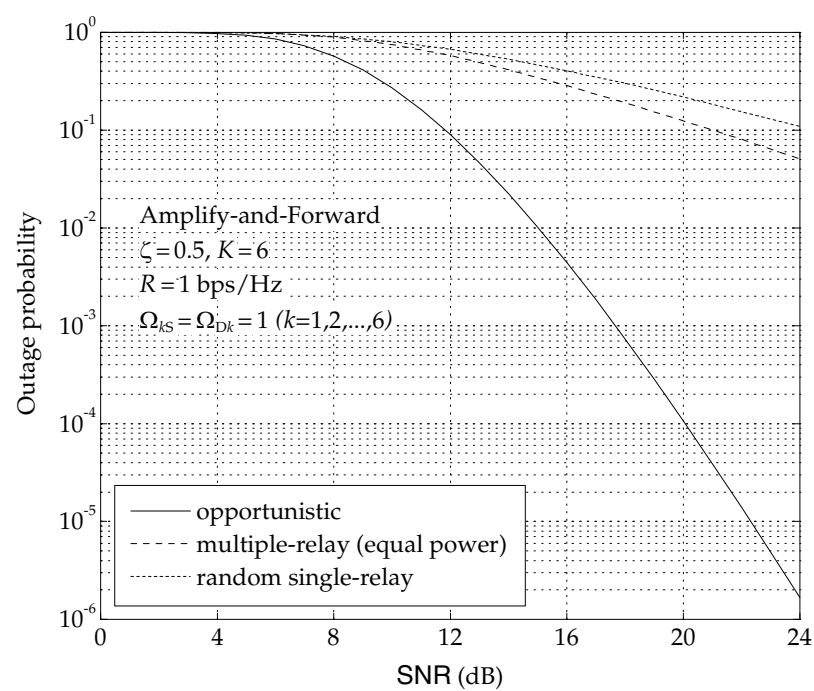

Fig. 3. Outage probability as a function of SNR for the AaF strategy with 6 relays $(K=6)$ at the end-to-end spectral efficiency $R=1$ $\mathrm{bps} / \mathrm{Hz}$ in symmetric channels with $\Omega_{\mathrm{S} k}=\Omega_{\mathrm{kD}}=1, k=1,2, \ldots, 6$. Opportunistic $\mathrm{AaF}$ relaying is compared with $\mathrm{AaF}$ schemes with equal-power MR transmissions and random single-relay selection.

in symmetric channels with $\Omega_{\mathrm{S} k}=\Omega_{k \mathrm{D}}=1, k=1,2, \ldots, 6$. As in Fig. 2, we set $\zeta=0.5$ due to no CSI at the source.

The opportunistic AaF relaying with a selection rule (27) is compared with $\mathrm{AaF}$ schemes with equal-power MR transmissions and random single-relay selection. Without global CSI at each relay, the optimal power allocation among $K$ relays subject to the aggregate power constraint (2) in MR AaF relaying is infeasible. In this case, equal-power allocation $\mathcal{P}_{k}=\mathcal{P}_{\text {relay }} / K, k=1,2, \ldots, K$, is again a possible approach. Under this limited channel knowledge, the opportunistic AaF relaying with the optimal single-relay selection has a considerable gain over equal-power MR AaF relaying, as seen from Fig. 3. It is also seen that choosing a random relay without a careful selection cannot provide a potential selection benefit from the opportunistic scheduling of relaying power.

\section{CONCLUSION}

Under the aggregate power constraint, we illustrated that cooperative relays can be useful even when they do not re-transmit but cooperatively listen, giving priority to the transmission of a single opportunistic relay. We showed the equivalence of the opportunistic DaF relaying (with the maxmin selection rule) to the outage bound of the optimal $\mathrm{DaF}$ strategy. We also presented opportunistic AaF relaying as the outage-optimal solution for single-relay selection and showed the significant gain over equal-power MR AaF relaying. Therefore, cooperation should be viewed not only as a transmission problem but also as a distributed relayselection task. Moreover, the opportunistic relaying requires no simultaneous same-frequency transmissions and its simplicity allowed implementation with existing low-complexity radio front ends [13].

\section{ACKNOWLEDGEMENT}

The authors would like to thank Ashish Khisti for his comments. This research was supported, in part, by the National Science Foundation under Grant CNS-0434816, the MIT Media Laboratory Digital Life Program, the Nortel Networks Graduate Fellowship Award, the Charles Stark Draper Laboratory Robust Distributed Sensor Networks Program, the Office of Naval Research Young Investigator Award N00014-03-10489, and the National Science Foundation under Grant ANI0335256.

\section{REFERENCES}

[1] J. H. Winters, "On the capacity of radio communication systems with diversity in Rayleigh fading environment," IEEE J. Select. Areas Commun., vol. 5, no. 5, pp. 871-878, June 1987.

[2] A. Sendonaris, E. Erkip, and B. Aazhang, "User cooperation diversityPart I: System description," IEEE Trans. Commun., vol. 51, no. 11, pp. 1927-1938, Nov. 2003.

[3] J. N. Laneman, D. N. C. Tse, and G. W. Wornell, "Cooperative diversity in wireless networks: Efficient protocols and outage behavior," IEEE Trans. Inform. Theory, vol. 50, no. 12, pp. 3062-3080, Dec. 2004.

[4] R. U. Nabar, H. Bölcskei, and F. W. Kneubühler, "Fading relay channels: Performance limits and space-time signal design," IEEE J. Select. Areas Commun., vol. 22, no. 6, pp. 1099-1109, Aug. 2004.

[5] G. Kramer, M. Gastpar, and P. Gupta, "Cooperative strategies and capacity theorems for relay networks," IEEE Trans. Inform. Theory, vol. 51, no. 9, pp. 3037-3063, Sept. 2005.

[6] M. Gastpar and M. Vetterli, "On the capacity of large gaussian relay networks," IEEE Trans. Inform. Theory, vol. 51, no. 3, pp. 765-779, Mar. 2005.

[7] J. N. Laneman and G. W. Wornell, "Distributed space-time coded protocols for exploiting cooperative diversity in wireless networks," IEEE Trans. Inform. Theory, vol. 59, pp. 2415-2525, Oct. 2003.

[8] P. Larsson and H. Rong, "Large-scale cooperative relay network with optimal coherent combining under aggregate relay power constraints," in Proc. Working Group 4, World Wireless Research Forum WWRF8 meeting, Beijing, China, 2004.

[9] I. Maric and R. Yates, "Forwarding strategies for gaussian parallel-relay networks," in Proc. Conf. on Inform. Sci. and Sys. (CISS'04), Princeton, New Jersey, Mar. 2004.

[10] M. Z. Win and J. H. Winters, "Methods and systems for spatial processing," U.S. Patent 6,804,312, Oct. 12, 2004.

[11] _ "Analysis of hybrid selection/maximal-ratio combining in Rayleigh fading," IEEE Trans. Commun., vol. 47, no. 12, pp. 17731776, Dec. 1999.

[12] A. F. Molisch and M. Z. Win, "MIMO systems with antenna selectionAn overview," IEEE Microwave Mag., vol. 5, no. 1, pp. 46-56, Mar. 2004.

[13] A. Bletsas, "Intelligent antenna sharing in cooperative diversity wireless networks," Ph.D. dissertation, Media Laboratory, Massachusetts Institute of Technology, Cambridge, MA, Sept. 2005.

[14] A. Bletsas, A. Khisti, D. P. Reed, and A. Lippman, "A simple cooperative diversity method based on network path selection," IEEE J. Select. Areas Commun., 2006, to be published.

[15] R. U. Nabar, H. Blcskei, and F. W. Kneubhler, "Fading relay channels: Performance limits and space-time signal design," IEEE J. Select. Areas Commun., vol. 22, no. 6, pp. 1099-1109, Aug. 2004.

[16] K. Azarian, H. E. Gamal, and P. Schniter, "On the achievable diversityvs-multiplexing tradeoff in cooperative channels," IEEE Trans. Inform. Theory, vol. 51, pp. 4152-4172, Dec. 2005.

[17] J. Adeane, M. R. D. Rodrigues, and I. J. Wassell, "Optimum power allocation in cooperative networks," in Proc. the Postgraduate Research Conference in Electronics, Photonics, Communications and Networks, and Computing Science, Lancaster, U.K., Mar.-Apr. 2005, pp. 23-24.

[18] H. Shin and M. Z. Win, "MIMO diversity in the presence of double scattering," IEEE Trans. Inform. Theory, 2005, submitted for publication. [Online]. Available: http://arxiv.org/abs/cs.IT/0511028 